\title{
1 Endopolyploidy as a potential driver of animal ecology and evolution
}

2 Maurine Neiman ${ }^{1}$, Margaret J. Beaton ${ }^{2}$, Dag O. Hessen ${ }^{3}$, Punidan D. Jeyasingh ${ }^{4}$, Lawrence J.

3 Weider $^{5}$

$4{ }^{1}$ Department of Biology, University of Iowa, Iowa City, IA, 52242, USA; maurine-

5 neiman@uiowa.edu

$6 \quad{ }^{2}$ Biology Department, Mount Allison University, Sackville, E4L 1G7, CANADA;

7 mbeaton@mta.ca

$8{ }^{3}$ Department of Biosciences, University of Oslo, Box 1066 Blindern, 0316 Oslo, NORWAY;

9 d.o.hessen@bio.uio.no

$10{ }^{4}$ Department of Integrative Biology, Oklahoma State University, Stillwater, OK, 74078, USA;

11 puni.jeyasingh@okstate.edu

$12{ }^{5}$ Department of Biology, Program in Ecology and Evolutionary Biology, University of

13 Oklahoma, Norman, OK, 73019,USA; ljweider@ou.edu 


\section{$14 \quad$ Abstract}

15 Endopolyploidy - the existence of higher-ploidy cells within organisms that are otherwise of a

16 lower ploidy level (generally diploid) - was discovered decades ago, but remains poorly studied

17 relative to other genomic phenomena, especially in animals. Our synthetic review suggests that

18 endopolyploidy is more common in animals than often recognized and likely influences a

19 number of fitness-related and ecologically important traits. In particular, we argue that

20 endopolyploidy is likely to play a central role in key traits such as gene expression, body and cell

21 size, and growth rate, and in a variety of cell types, including those responsible for tissue

22 regeneration, nutrient storage, and inducible anti-predator defenses. We also summarize evidence

23 for intraspecific genetic variation in endopolyploid levels and make the case that the existence of

24 this variation suggests that endopolyploid levels are likely to be heritable and thus a potential

25 target for natural selection. We then discuss why, in light of evident benefits of endopolyploidy,

26 animals remain primarily diploid. We conclude by highlighting key areas for future research

27 such as comprehensive evaluation of the heritability of endopolyploidy and the adaptive scope of

28 endopolyploid-related traits, the extent to which endopolyploid induction incurs costs, and

29 characterization of the relationships between environmental variability and endopolyploid levels.

31 Key words: Chromosomal evolution, genome evolution, endomitosis, endoreduplication,

32 endoreplication, phenotypic plasticity, ploidy level, polyploid, polyteny, somatic polyploidy 


\section{Contents:}

42 I. Introduction

43 II. What is endopolyploidy and how does it occur?

$44 \quad$ III. Where does endopolyploidy occur?

45 IV. Is endopolyploidy heritable?

46 V. Why endopolyploidy occurs: evolutionary and ecological drivers

47 a. Does endopolyploidy increase the level of gene expression?

48 b. Does endopolyploidy increase cell size?

49 c. Endopolyploidy and growth

d. Daphnia as a model system linking endopolyploidy, evolution, and ecology

\section{$57 \quad$ I. Introduction}

58 Genome size and structure often varies among and within eukaryotic species (Gregory, 2005;

59 Parfrey, Lahr \& Katz, 2008). From evolutionary and ecological perspectives, this variation is

60 significant because genomic features can influence growth rate, life cycle, metabolism,

61 morphology, and development (Gregory, 2005; Lynch, 2007; Parfrey et al., 2008; Hessen,

62 Daufresne \& Leinaas, 2013) and might also play a key role in divergence and speciation (Hessen

63 et al., 2013; Seehausen et al., 2014). Genome duplication (polyploidy) is widely acknowledged

64 as one of the most important sources of spontaneous genomic variation that can catalyze

65 phenotypic change and diversification (Soltis et al., 2014; Vanneste et al., 2014; Selmecki et al.,

66 2015). Here, we make the case that ploidy-level elevation within an individual (endopolyploidy)

67 might itself confer important evolutionary and ecological consequences, with a particular focus

68 on animals.

69

71 relative to the ancestral (usually diploid) state, is one of the most common and important means 
72 by which large-scale genomic variation is generated. Ploidy level can profoundly influence

73 molecular evolution, gene expression, and cellular or organismal phenotype (reviewed in King,

74 Seppälä \& Neiman, 2012; Mayfield-Jones et al., 2013; Neiman, Kay \& Krist, 2013a), and ploidy

75 elevation is thought to play an important role in the remarkably successful radiations of taxa such

76 as angiosperms (Soltis et al., 2009; Amborella Genome Project, 2013) and teleost fishes (Santini

77 et al., 2009). Despite the evident biological importance of ploidy level, there is no consensus on

78 the causes and consequences of ploidy level changes (Parisod, Holderegger \& Brochmann, 2010;

79 Mable, Alexandrou \& Taylor, 2011; Albertin \& Marullo, 2012; Leslie, 2014).

80

Ploidy is typically viewed as an organism-level trait. Although most multicellular

82 eukaryotes are diploid, it is increasingly clear that ploidy level variation is common across and

83 even within many plant and animal populations (Barlow, 1978; Mable et al., 2011). Less

84 recognized, especially in animals, is the fact that ploidy level variation is also common within

85 individuals (reviewed in Parfrey et al., 2008): even though the germline and most of the other

86 cells of any particular organism may be diploid (or triploid, tetraploid, etc.), certain tissues or a

87 subset of cells will very often feature a higher ploidy level than represented by the ploidy of the

88 organism as a whole. This phenomenon, known as endopolyploidy, is common in the embryonic

89 tissues of animals (trophoblast cells) (Lee, Davidson \& Duronio, 2009), and occurs in a variety

90 of other juvenile and adult animal tissues (Lee et al., 2009; Edgar, Zielke \& Gutierrez, 2014).

91 Endopolyploidy has also attracted attention as a central player in tumor development (Dewhurst

92 et al., 2014; Leslie, 2014).

93

94 The functional role of endopolyploidy is far from settled, but we will contend that it 
95 should not be dismissed as some cellular peculiarity of little evolutionary or ecological relevance

96 to animal populations. In particular, we will make the case that endopolyploidy is likely to be a

97 key contributor to a variety of ecologically important traits. More broadly, we will argue that

98 endopolyploidy is not only widespread, but also more important to animal evolution and ecology

99 than generally appreciated.

100

101 Critical insights into the evolutionary and ecological significance of endopolyploidy will

102 be revealed by: (i) determining the types of taxa and tissues that are typically associated with

103 endopolyploidy; (ii) identifying the cellular and organismal traits that are influenced by

104 endopolyploidy; and (iii), determining whether there is genetic variation in endopolyploidy

105 levels and/or inducibility that is visible to selection. We note that despite earlier papers

106 discussing the prevalence and highlighting the potential evolutionary and ecological relevance of

107 endopolyploidy (e.g., Nagl, 1976, 1978), there still do not exist enough data to allow rigorous

108 quantitative analyses. In this review, we synthesize recent insights and discoveries that both

109 illuminate the phenomenon of endopolyploidy and are consistent with the possibility that

110 endopolyploidy might have adaptive functions. Our ultimate motivation is to inspire new studies

111 directed towards revealing the ecological and evolutionary implications of endopolyploidy.

112

113 II. What is endopolyploidy and how does it occur?

114 To be clear, the term endopolyploidy (or endoreplication) has been used in the literature in both

115 broad and narrow contexts (in a manner similar to the use of the term heritability). As broadly

116 defined, endopolyploidy describes somatic cells with nuclei containing more than two times the

117 haploid DNA amount. This broad description does not preclude cells with under- or over- 
118 replication of specific genomic segments and includes both polyteny and the more narrowly

119 delineated endopolyploidy, which are the result of endocycling and endomitosis, respectively.

120 The increase in nuclear DNA amounts for all forms of the expansive endopolyploidy condition is

121 achieved during the $\mathrm{S}$ phase of altered cell cycles. Endocycling (polyteny) is the form of

122 endoreplication whereby chromosome strands are duplicated but mitosis is entirely bypassed,

123 leaving chromosome numbers unchanged (Edgar et al., 2014). By contrast, cells undergoing

124 endomitosis (endopolyploidy) fail to complete the late mitotic stages of telophase and/or

125 cytokinesis, resulting in duplicated chromosomes as discrete units within the same nucleus or in

126 separate nuclei and, typically, complete (unbiased) nuclear replication within a cell (Lee et al.,

127 2009). The number of endoreplication cycles (as endomitoses or endocycles) then determines the

128 ploidy level.

130 It is important to be clear about the differences between endopolyploidy and the related

131 but distinct phenomenon of polyploidy, which is defined as a condition where the ploidy level of

132 the majority of the cells in an organism (including the germline) is greater than diploid. Most

133 importantly, while endopolyploid cells arise from cells with lower ploidy via endoreplication, the

134 polyploid cells in polyploid organisms are generated from other polyploid cells by standard

135 mitotic processes. Endopolyploidy also differs from polyploidy by occurring within an otherwise

136 lower-ploidy organism and by its tissue-specific nature (cf. Comai, 2005). Despite these

137 differences, the many clear parallels between polyploidy and endopolyploidy mean that there is

138 obvious potential for insights generated from the study of polyploid organisms to apply to

139 endopolyploidy as well. 
142 Korpelainen et al., 1997) and a variety of densitometric methods (e.g., Rasch \& Wyngaard,

143 2008). Flow cytometry typically involves the automated measurement of large numbers of

144 fluorescently labeled cells. The primary advantages of flow cytometry are speed and the high

145 number of nuclei that can be processed at one time. Where flow cytometry falls short is with

146 respect to resolution, meaning that a flow cytometry approach is relatively likely to miss cells

147 that represent only a minor fraction of the population. DNA densitometry involves employing

148 microscopy and image analysis software on tissues subjected to the Feulgen reaction to quantify

149 the intensity of the nuclear stain for tissue-specific cells (see Hardie, Gregory \& Hebert, 2002

150 and Rasch, 2004 for relatively recent reviews of the protocol). While DNA densitometry is time

151 consuming, it is otherwise superior to flow cytometry in its ability to provide detailed ploidy

152 maps for individual tissues and detect ploidy levels that are rare within an organism (typically

153 the highest ploidy levels).

155 The developmental genetic mechanisms underlying endocycles and endomitosis are still

156 not fully understood and have been studied in detail only in a few model organisms (reviewed in

157 Edgar et al., 2014). Nevertheless, it is evident that endopolyploid tissues are more sensitive to

158 environmental stimuli such as nutrients and temperature than mitotic tissues (Wilson \& Roach,

159 2002). A good example of the sensitivity of induction of endopolyploidy to environmental

160 conditions was provided by Britton and Edgar (1998), who studied how starvation affected

161 proliferation in mitotic and endoreplicating cells in first-instar Drosophila larvae. They found

162 that while mitotic cells continued to proliferate in a nutrition-independent manner, most

163 endoreplicating cells instead entered a quiescent state under starvation, reinitiating division only 
164 when the starved larvae were again provided nutrients. Similar nutrient-dependent endocycle

165 responses have been observed in the ovarian nurse cells of Drosophila (Drummond-Barbosa \&

166 Spradling, 2001), mollusk neurons (Yamagishi et al., 2011), and the silk gland cells of

167 silkworms (Zhang et al., 2012). A recent study by Li et al. (2015) revealed that endomitotic

168 DNA synthesis in silk gland cells of silkworms fluctuated periodically, increasing during

169 intermolt stages when larvae feed and experiencing inhibition during molting periods when

170 larvae do not feed, also suggesting a close link between endopolyploidy and nutrition. A

171 mechanistic underpinning for this relationship is suggested by the evidence for covariation

172 between expression of cell cycle-related genes and synthesis of endomitotic DNA and the

173 discovery that key growth hormones such as ecdysone contribute to the regulation of endomitotic

174 DNA synthesis (Li et al., 2015). Effects of temperature on endoreplication and the degree of

175 endopolyploidy have been reported from dung flies (Blanckenhorn \& Llaurens, 2005),

176 Drosophila (Jalal et al., 2015), and Daphnia (Jalal et al., 2013). In all three of these examples,

177 individuals raised at lower temperatures exhibited a higher proportion of polyploid cells. This

178 demonstration of a connection between endopolyploidy and temperature is consistent with earlier

179 observations that polyploidy is more prevalent at low temperatures (e.g., Dufresne \& Hebert,

180 1995; Otto \& Whitton, 2000; Brochmann et al., 2004) and can be induced experimentally by

181 changes in temperature (e.g., Leggatt \& Iwama, 2003), though the extent to which the

182 temperature responses in endopolyploidy parallel those of induced polyploidy remains an open

183 question. Together, this growing body of literature highlights the potential for an important role

184 of endopolyploidy in phenotypic plasticity.

185

186 III. Where does endopolyploidy occur? 
187 Endopolyploidy has been documented in a diverse set of plant, fungal, and animal taxa (i.e.,

188 Nagl, 1978; Brodskiu \& Uryvaeva, 1985; Yin, Gater \& Karrer, 2010). One could argue that

189 "endopolyploidy" also exists in unicellular organisms, such as some bacteria, given the

190 documentation of extensive and variable polyploidization (i.e., multiple genome copies) in

191 different subfunctional regions of the cytoplasm of the relatively large (600 $\mu \mathrm{m}$ in length) single

192 cell bacterium Epulopiscium spp., a symbiont found in surgeonfish (Mendell et al., 2008). We

193 acknowledge that such examples from unicellular organisms do not fit neatly into the standard

194 definition of endopolyploidy (i.e., variation in ploidy level among cells or tissues within an

195 organism). Even so, it is worth considering the ecological and evolutionary mechanisms that

196 influence this type of genomic variation within unicellular organisms and the extent to which

197 these mechanisms are similar or different than the mechanisms that operate at the multicellular 198 level.

The evolution of endopolyploidy in eukaryotes may be quite ancient. In particular,

201 evidence for fundamental mechanistic similarities of endocycles across plant, fungal, and animal

202 taxa (e.g., down-regulation of cyclin-dependent kinase (CDK) while maintaining S-phase CDK;

203 Edgar et al., 2014) suggests that endopolyploidy might have first evolved in eukaryotes as long

204 as 800 million years ago (Edgar et al., 2014; but see discussion below of the likelihood of the

205 independent evolution of distinct molecular mechanisms leading to endopolyploidy).

Our survey of the animal taxa and the type and function of tissues in which

208 endopolyploidy has been observed demonstrates that endopolyploidy is widespread across

209 invertebrate (e.g., insects, crustaceans, annelids, mollusks) and vertebrate (e.g., fishes, birds, 
210 mammals) groups and occurs in many animal phyla and in a variety of tissues (Table 1). While

211 we do not intend this survey to provide a comprehensive report of the recorded instances of

212 endopolyploidy in animals, it does illustrate the taxonomic and functional expanse of the readily

213 available literature on the topic. In particular, our survey suggests that while substantial

214 information exists on endopolyploid levels in arthropods and mollusks and in selected tissues in

215 chordates, knowledge regarding the extent of endopolyploidy for many tissues and many animal 216 groups is limited to just one species or a few related taxa (Table 1).

218 Despite the remarkable diversity of taxa and tissues that feature endopolyploidy, the

219 cellular mechanisms that lead to endopolyploidy are broadly similar, featuring either alternating

220 S phases and $\mathrm{G}$ phases in the absence of mitosis or an abbreviated mitosis without completion of

221 cytokinesis (Lee et al., 2009; Edgar et al., 2014). At face value, these patterns might suggest that

222 the specific mechanisms underlying endopolyploidy are ancient and highly conserved,, although

223 the phylogenetic distribution of the various distinct molecular mechanisms leading to

224 endopolyploidy suggests that endopolyploidy has evolved independently on multiple occasions

225 in different taxa and different tissue types through evolutionary time (Anisimov, 2005; Anisimov

226 \& Zyumchenko, 2012; Edgar et al., 2014).

228 As has been previously shown in plants (Nagl, 1978; Barow \& Meister, 2003; Edgar et

229 al., 2014), endopolyploid levels in animals also feature taxon- and tissue-specific variation

230 (Table 1). In at least some invertebrates, a large fraction of somatic cells may be polyploid

231 (Scholes et al., 2014), although the degree of endopolyploidy can itself be influenced by internal

232 (e.g., age, nutritional status) and external (e.g., temperature) environmental factors (e.g., Beaton 
233 \& Hebert, 1997; Korpelainen, Ketola \& Hietala, 1997; Yamagishi et al., 2011; Jalal et al., 2013).

234 Among vertebrates, hepatocytes and cardiomyocytes can be mono- or binucleate, but the highest

235 recorded level for either tissue in these animals is $32 \mathrm{C}$ (Table 1). The insect fat body, which,

236 similar to the vertebrate liver, performs multiple functions related to metabolism and storage,

237 also exhibits low to moderate endopolyploid levels for a hemipteran (i.e., a maximum of 128C;

238 Nagl, 1978). By contrast, mammalian trophoblast cells can exhibit ploidy levels of 64-4096C

239 (Nagl, 1978; Anatskaya, Vinogradov \& Kudryavtsev, 1994; Vinogradov, Anatskaya \&

240 Kudryavtsev, 2001; Anatskaya \& Vinogradov, 2004). In arthropods (e.g., hymenopterans),

241 endopolyploid levels can reach 512C across tissues such as Malpighian tubules, small intestine,

242 and thoracic gland (Nagl, 1978; Yamagishi et al., 2011), and salivary glands routinely achieve

243 endopolyploid levels of $1024 \mathrm{C}$ or more (Nagl, 1978). The neurons of mollusks feature

244 remarkable ploidy variation, from a modest $32 \mathrm{C}$ in the land snail Triodopsis divesta to an

245 astounding 200000C in the gigantic neurons of the sea hare Aplysia californica (Lasek \& Dower,

246 1971; Mandrioli et al., 2010). The highest endopolyploid level that has been recorded in any

247 animal is $>500000 \mathrm{C}$, reported from the silk-producing glands of the silk moth Bombyx mori

248 (Perdrix-Gillot, 1979; Gregory \& Hebert, 1999). In general, the maximal tissue-specific ploidy

249 level achieved via endopolyploidy appears to be developmentally programmed (Edgar et al.,

250 2014), but it is still not clear what governs maximal endopolyploid levels in different tissues and

251 taxa.

252

253 IV. Is endopolyploidy heritable?

254 Individual-level heritable phenotypic variation (i.e., either broad-sense heritability, $\mathrm{H}^{2}$, or

255 narrow-sense heritability, $\mathrm{h}^{2},>0$ ) is the raw material for evolution by natural selection, raising 
256 the questions of 1) whether there exists among-individual variation in endopolyploidy levels

257 and/or induction thresholds, and 2) whether this variation is heritable. While there are relatively

258 few studies of endopolyploidy that use the quantitative genetics approach required to estimate

259 heritability, both of these questions have been addressed indirectly by the multiple studies that

260 provide empirical evidence for consistent intraspecific differences in levels of endopolyploidy

261 among distinct lineages and genotypes (Beaton \& Hebert, 1997; Korpelainen et al., 1997;

262 Cheniclet et al., 2005; Gegas et al., 2014). In other words, these studies demonstrate a critical

263 component of heritability: that phenotypic differences in endopolyploid levels are reliably

264 transmitted to offspring.

266 Some of the best examples of such intraspecific variation in animals are provided by the

267 freshwater microcrustacean Daphnia (Fig. 1; also see a similar example from other Daphnia

268 species in Beaton \& Hebert, 1989). For example, Korpelainen et al. (1997) found that the

269 percentage of $2 \mathrm{C}, 4 \mathrm{C}$, and $8 \mathrm{C}$ cells ranged from $\sim 63-80 \%, \sim 18-32 \%$, and $\sim 2-5 \%$ of all cells,

270 respectively, among Daphnia genotypes isolated from 13 different Finnish rockpool populations.

271 Similarly, Beaton and Hebert (1997) noted extensive interspecific variation in the number of

272 polyploid cells located in the head/helmet region of 20 daphniid species as well as substantial

273 intraspecific variation in this trait among genotypes within species. The existence of both

274 genotype- and species-specific endopolyploid levels in Daphnia suggests that endopolyploid

275 levels have at least a partial genetic basis and thus are potentially heritable.

277 As is typical for studies on any aspect of endopolyploidy, there is a larger body of

278 evidence from plants than from animals in support of the possibility that endopolyploid 
279 phenotypes can be heritable. One clear example is provided by Cheniclet et al. (2005), who

280 examined across-line levels of endopolyploidy in the pericarp of the fruit of tomato (Solanum

281 lycopersicum). This study revealed extensive significant across-line variation (i.e., genetic

282 variation) for the extent of expression of the endopolyploid phenotype as well as strong positive

283 correlations between endopolyploid levels and cell diameter and fruit weight in S. lycopersicon.

284 Intraspecific genetic variability in tissue-specific endopolyploidy has also been demonstrated in

285 other plant taxa (e.g., accessions of Arabidopsis thaliana; Gegas et al., 2014). Altogether, there

286 is a growing body of data indicating that the intraspecific variation required for endopolyploid

287 levels to be heritable exists. The critical next step towards evaluating whether natural selection

288 plays a role in maintaining variation in endopolyploid levels across tissues and taxa-

289 determining whether endopolyploidy levels and induction thresholds can evolve via selection on

290 endopolyploid-associated phenotypes - remains to be empirically addressed.

292 V. Why endopolyploidy occurs: evolutionary and ecological drivers

293 Here, we synthesize concepts and data to address the extent to which endopolyploidy is likely to

294 influence evolutionary and ecological processes, and in particular, evaluate whether

295 endopolyploidy might serve an adaptive function (Table 1, Fig. 1). Most of the examples that we

296 discuss invoke or assume associations between endopolyploidy and two fundamentally important

297 cell-level characteristics, (1) levels of gene expression, and (2), cell size. Because these cellular

298 traits comprise plausible links between endopolyploidy and organism-level traits (e.g., body size,

299 growth rate) that are likely themselves to often influence fitness-related phenotypes in animals,

300 we then summarize and synthesize the data that allow us to address these potential links between

301 endopolyploidy and organismal biology. In particular, we focus on whether and to what extent 
302 endopolyploidy influences gene expression and cell size and whether these functional

303 connections may have evolutionary and/or ecological consequences, particularly with respect to

304 organ or organismal growth. Finally, we ask why, in light of apparent evolutionary and

305 ecological advantages of endopolyploidy, most cells in most animals remain diploid.

a. Does endopolyploidy increase the level of gene expression?

308 It is commonly assumed that endopolyploidy functions to generate the extra gene copies needed

309 to produce the RNA required to sustain key fitness-enhancing anabolic (e.g., protein synthesis)

310 and/or catabolic (e.g., energy metabolism) processes. This predicted functional connection

311 between endopolyploidy and levels of gene expression is nearly always followed by the caveat

312 that whether endopolyploidy in fact influences transcription remains unclear (Edgar \& Orr-

313 Weaver, 2001; Leiva-Neto et al., 2004; John \& Qi, 2008; Lee et al., 2009; Bourdon et al., 2010;

314 Chevalier et al., 2011; Mayfield-Jones et al., 2013; Sher et al., 2013), to the extent that Bourdon

315 et al. (2010) concluded that the hypothesis that a major functional role of endopolyploidy is to

316 increase gene expression had yet to be adequately tested. Subsequent research by Bourdon et al.

317 (2012) in tomato showed that ribosomal RNA, RNA polymerase II, and gene transcript levels

318 increase with nuclear ploidy level, providing direct evidence for a positive relationship between

319 endopolyploidy and gene expression in a vascular plant model system. Determining whether

320 these results extend to animals requires similar rigorous assessments in animal systems.

322 A promising starting point for addressing questions regarding a functional role for

323 endopolyploidy as a mechanism to increase gene expression and protein production in animals is

324 provided by silk-producing arthropods such as spiders, silk moths, and some caddisflies 
325 (Trichoptera). These animals are ideal models to explore such links because 1) their silk-

326 producing glands typically consist of polyploid cells (Sehnal \& Sutherland, 2008), 2), silk is a

327 very conspicuous protein product that clearly may be the target of selection, and 3), there exist

328 species that only produce silk during a single life stage as well as species that use silk throughout

329 their life cycle, enabling powerful across-taxa comparisons. The common occurrence of

330 endopolyploidy in animal silk (and venom) glands led Gregory and Shorthouse (2003; also see

331 Rasch \& Connelly, 2005) to suggest that there very likely is an association between high protein

332 output and endopolyploidy in such glands, and that a comparison among species with different

333 silk-spinning habits would be rewarding in this context. One of the more striking examples of a

334 positive endopolyploid level-silk production relationship is provided by the silk moth Bombyx

335 mori, whose silk-producing glands feature endopolyploid levels exceeding 500000C (Perdrix-

336 Gillot, 1979; Gregory \& Hebert, 1999), likely linked to intensive artificial selection for silk yield

337 (Perdrix-Gillot, 1979). Recent evidence that the genes involved in silk production in B. mori

338 have experienced rapid evolution since these moths were domesticated (Xia et al., 2009),

339 coupled with the likely possibility of a causal endopolyploidy-silk production connection,

340 provide another line of evidence that tissue-specific endopolyploid levels are evolvable.

342 b. Does endopolyploidy increase cell size?

343 There is often (e.g., Melaragno, Mehrotra \& Coleman, 1993; Cheniclet et al., 2005; Gonzalez et

344 al., 2010; Bourdon et al., 2010; recently reviewed in De Veylder, Larkin \& Schnittger, 2011;

345 Edgar et al., 2014) but not always (Fankhauser, 1945; Bourdon et al., 2010; De Veylder et al.,

346 2011) a positive association between nuclear ploidy level and cell size in both plants and

347 animals. While the precise mechanisms that link endopolyploidy to increased cell size remain 
348 unclear (John \& Qi, 2008; Bourdon et al., 2010; De Veylder et al., 2011), one possibility is that

349 the increased DNA content in the nuclei of polyploid cells results in increased nuclear volume,

350 which itself then induces increased cell volume ("karyoplasmic ratio"; Cavalier-Smith, 1982;

351 Olmo, 1983; Sugimoto-Shirasu \& Roberts, 2003; Cheniclet et al., 2005; Bourdon et al., 2010;

352 Gonzalez et al., 2010). This hypothesis has found recent direct support in a study of the

353 relationship between endopolyploidy, cell size, and nuclear size in tomato (Bourdon et al., 2012).

354 A contrary view is expressed by John and Qi (2008; also see e.g., Massonnet et al., 2011; Gegas

355 et al., 2014), who argue that recent evidence that increases in cell size are required for the

356 initiation of endoreplication suggests that at least in some instances, endopolyploidy might be

357 more accurately considered an effect rather than a cause of increased cell size.

359 Regardless of the mechanisms connecting endopolyploidy to cell size, it is evident that

360 increased cell size can affect traits that might influence organismal ecology and/or fitness (Olmo,

361 1983; Szaro \& Tompkins, 1987). These connections between cell size and phenotype are often

362 mediated by relationships between cell size, cell number, and/or body size, which themselves are

363 quite different in plants than in animals (Sugimoto-Shirasu \& Roberts, 2003). For example, while

364 polyploid plants frequently have both larger cells and larger bodies than diploid counterparts, the

365 relatively large cells that characterize polyploid vs. diploid forms of particular animals often

366 (Day \& Lawrence, 2000; e.g., Fankhauser, 1945; Santamaria, 1983; Henery, Bard \& Kaufman,

367 1992) but not always (e.g., Hessen et al., 2013) lead to larger body sizes. An excellent example

368 of the complex consequences of ploidy elevation in animals is provided by polyploid

369 salamanders, which have larger but fewer cells than diploid counterparts (e.g., Fankhauser,

370 1945). This loss of cell number does appear to confer costs related to organ complexity: 
371 polyploid salamanders have fewer neurons and simpler brains than their diploid counterparts

372 (Roth, Blanke \& Wake, 1995; also see Roth et al. 1994). Vernon and Butsch (1957) even argued

373 that these differences in neuron number and brain structure could underlie the inferior

374 performance of tetraploid vs. diploid salamanders in a maze running experiment.

376 c. Endopolyploidy and growth

377 It is evident that endopolyploidy has the potential to affect traits (e.g., gene expression levels,

378 cell and body size, organ complexity, behavior) that might confer ecological and/or fitness

379 consequences. In particular, the connections between endopolyploidy and traits that directly or

380 indirectly influence gene expression and cell and/or body size suggest that an important

381 evolutionary and ecological function of endopolyploidy might be to facilitate organ or

382 organismal growth in conditions where early maturation, large size, or rapid growth/regeneration

383 are favored (Cavalier-Smith, 1978; Melaragno et al., 1993; Anatskaya et al., 1994; e.g., Scholes

384 \& Paige, 2011, Losick, Fox \& Spradling, 2013).

385

386 Animals can grow either by increasing their cell number or by increasing their cell size.

387 For organisms with fixed cell numbers (e.g., nematodes), growth is largely attributed to the

388 increased cell size associated with endopolyploidy (Flemming et al., 2000; Edgar \& Orr-Weaver,

389 2001; Lozano et al., 2006). While this form of whole-body growth is thought to be relatively

390 uncommon (Day \& Lawrence, 2000), it is probably more widespread than hitherto recognized

391 because it has been observed in a diverse set of invertebrate taxa (e.g., appendicularians, Ganot

392 \& Thompson, 2002; copepods, Rasch \& Wyngaard, 2008). Under environmental conditions that

393 inhibit cell division (e.g., desiccation, UV-B irradiation), increases in cell size that are correlated 
394 with endopolyploidy might even provide a mechanism by which organ/organism size can be

395 maintained in the absence of cell division (Sugimoto-Shirasu \& Roberts, 2003; De Veylder et al.,

396 2011; Gegas et al., 2014).

397

The silk-producing moth Ephestia küehniella provides a striking example of how

399 endopolyploidy can regulate growth of specific organs during ontogeny. Between the first and

400 second larval instars, the cells comprising the Malphigian tubules and silk glands increase in

401 volume by factors of $\sim 1800$ and 3100 , respectively, via repeated endocycles. By the final larval

402 instar, the Malphigian tubules have reached $1024 \mathrm{C}$, while the silk glands have attained up to

403 8192C (Buntrock et al., 2012). Another line of evidence connecting endopolyploidy and organ

404 growth in E. küehniella is provided by evidence that the size of the scales covering E. küehniella

405 wings is positively associated with the endopolyploid level of the epidermal cell beneath the

406 scale: 8C cells tend to be found below relatively small scales, and the largest scales often are

407 coupled with 32C cells (Kühn, 1965, as cited in Nagl, 1978).

Two recent studies of inter- and intra-individual variation in endopolyploidy in several

410 ant species illustrate how endopolyploidy may be related both to body size and organ function

411 (Scholes, Suarez \& Paige, 2013; Scholes et al., 2014). Scholes et al. (2013) found that body size

412 is positively related to endopolyploidy, such that larger workers have relatively high levels of

413 endopolyploidy across a variety of tissues. The authors discovered that abdominal tissues had the

414 highest endopolyploid levels of all, inspiring Scholes et al. (2014) to characterize endopolyploid

415 levels in various organs of the giant ant Dinoponera australis. This study revealed significantly

416 higher levels of endopolyploidy in organs involved in digestion (e.g., foregut/crop, mid-gut) and 
417 exocrine function (e.g., Dufour's gland - pheromone production) relative to tissues/organs in

418 either the head or the thorax, which themselves did not differ significantly from one another in

419 mean endopolyploid levels. The one exception to this pattern was the mandibular gland of the

420 exocrine system. Although this gland resides in the head, it also exhibited high endopolyploid

421 levels, indicating that there is an elevated level of endopolyploidy for the exocrine system even

422 when tissue source is taken into account. Scholes et al. (2014) interpreted this result as

423 representing a possible connection between elevated endopolyploidy in tissues that require high

424 cellular metabolism and specialized function (also see Anatskaya et al., 1994).

425

426 d. Daphnia as a model system linking endopolyploidy, evolution, and ecology.

427 Daphnia species are well suited as an animal model for studies of endopolyploidy because of 428 widespread tissue involvement (Fig. 1) and the diversity of associated functions. The

429 increasingly prominent role of Daphnia as a model organism for functional genomics (Colbourne 430 et al., 2011) allows for a thorough evaluation of gene regulation, expression, and dosage effects

431 at the tissue (or cellular) level. Polyploid cell numbers for a given tissue appear to be established

432 by the first instar (Beaton \& Hebert, 1999), pointing to embryogenesis as the transitional period

433 for the development of endopolyploidy in Daphnia.

435 The drivers of maximum ploidy levels in each Daphnia tissue seem to vary. For example, 436 epipodites, key ion regulatory tissues (Kikuchi, 1983), are entirely polyploid (Fig. 1), which may

437 reflect ontological changes in sodium uptake mechanisms (Bianchini \& Wood, 2008) and/or

438 function to reduce cell-cell interactions in the tissue. Ploidy levels in the epipodites plateau soon

439 after reaching maturity, indicating tight developmental control (Beaton \& Hebert, 1999). 
440 Because the animal continues to grow throughout life but the endopolyploid level in the

441 epipodites stays constant, the osmoregulatory load per cell may increase over time. By contrast,

442 cells in the tissues associated with food acquisition (e.g., secretory labrum, lipid storage cells) are

443 not entirely polyploid, but the endopolyploid cells in these tissues have the highest ploidy levels

444 (2048C) found in the animal as a whole (Sterba, 1956, 1957; Beaton \& Hebert, 1999). Again, in

445 contrast to the epipodites, in which endopolyploid levels stabilize by maturity, the initiation and

446 number of endomitotic cycles in tissues associated with food acquisition are linked to

447 development, growth, and nutritional status (Beaton \& Hebert, 1999).

Daphnia produce a variety of inducible epidermal structures (e.g., neckteeth, spines,

450 helmets) in response to chemical signals indicating the presence of predators (Brooks, 1965).

451 These defensive structures form as modifications of the epidermis, a primarily diploid tissue

452 containing occasional polyploid cells at the dorsal and ventral margins (Fig. 1). Beaton and

453 Hebert (1997) proposed a regulatory function for the polyploid epidermal cells wherein these

454 cells modulate surrounding cell division and allow localized tissue growth via the release of an

455 unknown mitogen (Beaton \& Hebert, 1997). In a preliminary loss-of-function study, the ablation

456 of selected cephalic polyploid cells in D. lumholtzi resulted in a helmet size reduction of $\sim 20 \%$ -

$45740 \%$ after one molt, supporting this model (Beaton, unpubl). Recently, Weiss et al. (2012)

458 showed that polyploid cells in the head epidermis of several species of Daphnia have plasma

459 bulges and high rates of protein synthesis. Since then, an immunohistochemistry-based study

460 revealed that these cells serve as storage sites for dopamine, a neurohormone (L. Weiss, pers.

461 comm.). While the mechanism of action for dopamine will depend on the receptor type upon

462 which it acts, dopamine is known to act as modulator of stress responses in insects (Johnson \& 
463 White, 2009), lending further support to the polyploid control center model proposed by Beaton

464 and Hebert (1997). A preliminary survey of gene expression in juvenile D. lumholtzi raised in the

465 presence or absence of well-fed fish revealed that predator-induced animals (with helmets double

466 the size of control animals at similar body sizes) exhibited a general down-regulation of mRNA

467 transcripts relative to Daphnia in the predator-free treatments (McKinnon, 2013). This result

468 hints at an alternative (though non-mutually exclusive) hypothesis for the functional role of

469 endopolyploidy in this tissue: Because neonates, regardless of stress level, form helmets (though

470 these helmets are much smaller than those produced by stressed adults), perhaps helmet

471 formation is the default state and, in the absence of predator cues, enlarged head cells negatively

472 modulate cell division. When faced with predation risk, the transcriptional activity of these

473 polyploid cells decreases, allowing uninhibited cell division (and maximal helmet formation).

474 Regardless of the mechanism involved, the presence of endopolyploidy in epidermal tissue

475 appears to be critical in reducing vulnerability to predation through the production of inducible

476 defenses in Daphnia.

$478 \quad$ VI. Why aren't all animal cells polyploid?

479 We here have summarized evidence demonstrating that endopolyploidy is very widely

480 distributed across animal taxa and tissues and is likely to often confer substantial advantages.

481 Even so, and even in organisms harboring a relatively high fraction of polyploid cells, most cells

482 remain diploid, which suggests that there may exist substantial costs associated with

483 endopolyploidy.

485 One possible cost associated with endopolyploidy was suggested by Melaragno et al.

486 (1993), who speculated that once a cell begins cycling endomitotically, it cannot return to the 
487 mitotic cycle and cannot thus create additional new cells (also see Edgar \& Orr-Weaver, 2001;

488 John \& Qi, 2008). This hypothesis is supported by data from Arabidopsis indicating that new

489 cells are primarily produced by diploid progenitors (Galbraith, Harkins \& Knapp, 1991), though

490 exceptions have been reported in at least three invertebrate species (Beaton \& Hebert, 1999; Fox,

491 Gall \& Spradling, 2010).

492

493 There is some evidence that larger genome and/or cell size can slow the rate of cell

494 division (reviewed in Gregory, 2005), suggesting the non-mutually exclusive possibility that

495 polyploid cells might generate costs associated with a relatively low cell division rate. In

496 addition, the generally positive relationship between endopolyploid level and cell size (as

497 described above) will also reduce the cell surface area to volume ratio, potentially generating

498 constraints on the efficiency of energy, nutrient, and waste transport between cells and

499 intercellular space (Gregory, 2005). The lack of data on the abundance and distribution of

500 organelles and surface transport systems in endopolyploid cells compared to mitotic cells

501 precludes any conclusive arguments about such potential costs of endopolyploidy but should be a

502 fruitful avenue for future research.

504 There are also potential material costs associated with higher cellular DNA content that

505 are themselves connected to the notable abundance of nitrogen $(\mathrm{N})$ and phosphorus $(\mathrm{P})$ in nucleic

506 acids. The nucleus contains a relatively large fraction of nucleic acids, and is thus rich in $\mathrm{P}$ (ca.

$5072.5 \%$ P per dry weight (DW)). Chromosomes are nearly $4 \% \mathrm{P}$ per DW and $>15 \% \mathrm{~N}$, while

508 DNA and RNA are the most P-rich macromolecules in the cell, with $>5 \% \mathrm{P}$ of DW (Sterner \&

509 Elser, 2002). An especially large fraction of $\mathrm{P}$ is bound in nucleic acids in unicellular 
510 heterotrophic eukaryotes and invertebrates (Sterner \& Elser, 2002). Hessen et al. (2010)

511 hypothesized that because $\mathrm{P}$ is often scarce in nature, reallocation of P from DNA to RNA in

512 these organisms via genome downsizing could constitute an evolutionary response to selection

513 favoring increased individual growth rate.

514

515 These connections between P investment in DNA vs. RNA and organismal growth rate

516 imply that there could also be material costs of endopolyploidy related to the $\mathrm{P}$ allocation

517 demanded by polyploid tissue. Indeed, Neiman et al., (2009) found that polyploid snails had

518 higher per unit mass P content than diploid counterparts, indicating that higher ploidy levels

519 might bear material costs. Furthermore, evidence for connections between organismal growth

520 rates, P availability, and ploidy level in snails (Neiman, Kay \& Krist, 2013b) and vascular plants

521 (Šmarda et al., 2013) do suggest a tradeoff between the higher rate of transcription and

522 production that could be afforded by ploidy elevation and the metabolic and/or nutrient costs

523 associated with a higher rate of synthesis of body components. These results highlight the

524 likelihood that ploidy elevation (and, perhaps, endopolyploidy) is more likely to confer

525 advantages in conditions where the availability of resources (e.g., phosphorus, Hessen et al.,

526 2010; Neiman et al., 2013a) needed to synthesize more/larger tissues is relatively high (also see

527 Mayfield-Jones et al., 2013). Such mechanisms could play an important role in the evolutionary

528 responses of populations to drastic alterations to environmental nutrient availability caused by

529 anthropogenic activities.

530

$531 \quad$ VII. Conclusions and future directions 
532 (1) It is evident that endopolyploidy is both common and is often associated with major

533 phenotypic consequences, though this phenomenon remains relatively understudied in animals.

534 Some of these consequences of endopolyploidy (e.g., response to herbivory, wound healing, the

535 induction and formation of morphological defenses) have either been documented (e.g., Beaton

536 \& Hebert, 1997; Scholes \& Paige, 2011; Bainard et al., 2012; Losick et al., 2013; Scholes \&

537 Paige, 2014) or are likely to serve as potential drivers of ecological and evolutionary processes.

(2) Because relatively little empirical attention has been directed to the study of

540 endopolyploidy in evolutionary and ecological contexts, especially in animals, critical questions

541 regarding the importance of endopolyploidy for animal evolution and ecology remain

542 unanswered, ranging from the evolutionary processes underlying the complex phylogenetic

543 distribution of endopolyploidy to the molecular basis of endocycling and endomitosis.

544 Quantification of the frequency and distribution of endopolyploidy across tissues, organisms, and

545 different environmental conditions will allow for rigorous characterization of patterns at

546 physiological, phylogenetic, and ecological levels. These data can be used to perform a wide

547 variety of important tests of the evolutionary and ecological significance of polyploidy. Such

548 tests would range from addressing whether there are phylogenetic patterns in the incidence of

549 endopolyploidy (e.g., Anisomov \& Zyumchenko, 2012) and whether there exist specific

550 ecological "syndromes" (i.e., terrestrial, marine, freshwater) that might favor the evolution of

551 endopolyploidy, to determining whether endopolyploidy is more prevalent in secretory tissues

552 (e.g., Perdrix-Gillot, 1979) and/or rapidly growing tissues (e.g., Anatskaya \& Vinogradov,

553 2002)? A powerful empirical approach in this context would be to compare sister taxa that show

554 distinct differences in the incidence of endopolyploidy, with the goal of identifying the 
555 ecological and/or evolutionary factors involved in these differences. The availability of these

556 data will catalyze formulation of hypotheses about the proximate (e.g., Edgar et al., 2014) and

557 ultimate (e.g., Scholes \& Paige, 2014) mechanisms that underlie the induction and extent of

558 endopolyploidy.

560 (3) In particular, quantification of the heritability of endopolyploidy levels and

561 inducibility and evaluation of whether endopolyploid levels respond to selection on phenotypes

562 connected to endopolyploidy (e.g., cell size, protein production, organ growth rate) will provide

563 important tests of the extent to which endopolyploidy is likely to be a major player in adaptive

564 evolution. An important role for endopolyploidy as a driver of evolutionary processes will

565 require that endopolyploidy levels and inducibility thresholds are heritable and can influence

566 organismal fitness. Key research directions from an ecological perspective, which are connected

567 to but distinct from the evolutionary side of the story, include the evaluation of associations

568 between environmental variability (e.g., nutrient availability, predator presence) and

569 endopolyploidy and the extent to which endopolyploid induction incurs costs. Empirical studies

570 of whether and how particular environmental conditions can induce endopolyploidy and how the

571 induction of endopolyploidy affects ecologically relevant traits like sensitivity to nutrient

572 limitation and susceptibility to predation will provide important steps towards establishing the

573 extent to which endopolyploidy influences ecology, and vice versa, in natural animal

574 populations.

575

576 (4) Definitive answers to such fundamental questions about the evolution and ecology of

577 endopolyploidy will require an interdisciplinary approach. In particular, ecologists, geneticists, 
578 developmental biologists, physiologists, and evolutionary biologists will need to work together

579 to evaluate the ecological stimuli for endopolyploid induction, how endopolyploidy influences

580 fundamental cell-, tissue-, and organism-level traits like cell and organ size, gene expression, and

581 growth rate, and in turn, how these traits influence organismal and population ecology and

582 evolution.

583

584 (5) Our ultimate goal would be to understand how these traits impact ecological functions

585 and the adaptive potential of natural populations.

586

$587 \quad$ VIII. Acknowledgments

588 MN was funded by the National Science Foundation grant MCB-1122176. This work also

589 benefited from the GENOME research project supported by The Research Council of Norway,

590 grant No. 1964.

591

$592 \underline{\text { IX. References }}$

593 Albertin, W. \& Marullo, P. (2012). Polyploidy in fungi: evolution after whole-genome

$594 \quad$ duplication. Proceedings of the Royal Society of London B 279, 2497-2509.

595 Amborella Genome Project. (2013). The Amborella genome and the evolution of flowering

596 plants. Science 342, DOI: 10.1126/science.1241089

597 Anatskaya, O. V., \& Vinogradov, A. E. (2002). Myocyte ploidy in heart chambers of birds with

598 different locomotor activity. Journal of Experimental Zoology 293, 427-441. 
599

600

601

602

603

604

605

606

607

608

609

610

611

612

613

614

615

616

617

618

619

620

Anatskaya, O. V. \& Vinogradov, A. E. (2004). Heart and liver as developmental bottlenecks of mammal design: evidence from cell polyploidization. Biological Journal of the Linnean Society 83, 175-186.

Anatskaya, O. V., Vinogradov, A. E. \& Kudryavtsev, B. N. (1994). Hepatocyte polyploidy and metabolism/life-history traits: hypotheses testing. Journal of Theoretical Biology $\mathbf{1 6 8}$, 191-199.

Anisimov, A. P. (2005). Endopolyploidy as a morphogenetic factor of development. Cell Biology International 29, 993-1004.

Anisimov, A. P. \& Zyumchenko, N. E. (2012). Evolutionary regularities of development of somatic polyploidy in salivary glands of gastropod mollusks: V. Subclasses Opisthobranchia and Pulmonata. Cell and Tissue Biology 6, 268-279.

Bainard, J. D. \& Newmaster, S. G. (2010). Endopolyploidy in bryophytes: widespread in mosses and absent in liverworts. Journal of Botany 2010, 316356.

Bainard, J. D., Bainard, L. D., Henry, T. A., Fazekas, A. J. \& Newmaster, S. G. (2012). A multivariate analysis of variation in genome size and endoreduplication in angiosperms reveals strong phylogenetic signal and association with phenotypic traits. New Phytologist 196, 1240-1250.

Barlow, P. W. (1978). Endopolyploidy: Towards an understanding of its biological significance. Acta Biotheoretica 27, 1-18.

Barow, M. \& Meister, A. (2003). Endopolyploidy in seed plants is differently correlated to systematics, organ, life strategy and genome size. Plant, Cell \& Environment 26, 571584. 
621 Beaton, M. J. \& Hebert, P. D. N. (1989). Miniature genomes and endopolyploidy in 622 cladoceran crustaceans. Genome 32, 1048-1053.

623 Beaton, M. J. \& Hebert, P. D. N. (1997). The cellular basis of divergent head 624 morphologies in Daphnia. Limnology and Oceanography 42, 346-356.

625 Beaton, M. J. \& Hebert, P. D. N. (1999). Shifts in postembryonic somatic ploidy levels 626 in Daphnia pulex. Hydrobiologia 394, 29-39.

627 Bianchini, A. \& Wood, C. W. (2008). Sodium uptake in different life stages of crustaceans: the 628 water flea Daphnia magna Strauss. Journal of Experimental Biology 211, 539-547.

629 Blanckenhorn, W. \& Llaurens, V. (2005). Effects of temperature on cell size and number in the 630 yellow dung fly Scathophaga stercoraria. Journal of Thermal Biology 30, 213-219.

631 Blow, J. J. \& Hodgson, B. (2002). Replication licensing — Origin licensing: defining 632 the proliferative state? Trends in Cell Biology 12, 72-78.

633 Bourdon, M., Frangne, N., Mathieu-Rivet, E., Nafati, M., Cheniclet, C., Renaudin, J.P. \& 634 Chevalier, C. (2010). Endoreduplication and growth of fleshy fruits. Progress in Botany $635 \quad \mathbf{7 1}, 101-132$.

636 Bourdon, M., Pirrello, J., Cheniclet, C., Coriton, O., Bourge, M., Brown, S., Moïse, A., 637 Peypelut, M., Rouyère, V., Renaudin, J.-P., Chevalier, C. \& Frangne, N. (2012).

638 Evidence for karyoplasmic homeostasis during endoreduplication and a ploidy-dependent 639 increase in gene transcription during tomato fruit growth. Development 139, 3817-3826.

640 Britton, J. S. \& Edgar, B. A. (1998). Environmental control of the cell cycle in

641 Drosophila: nutrition activates mitotic and endoreplicative cells by distinct mechanisms.

642 Development 125, 2149-2158. 
643 Brochmann, C., Brysting, A. K., Alsos, I. G., Borgen, L., Grundt, H. H., Scheen, A.-C. \& Elven, 644 R. (2004). Polyploidy in arctic plants. Biological Journal of the Linnean Society 82, 521$645 \quad 536$.

646 Brodskiĭ, V. I. \& Uryvaeva, I. V. (1985). Genome multiplication in growth and 647 development: biology of polyploid and polytene cells (Vol. 15). Cambridge University $648 \quad$ Press, Cambridge.

649 Brooks, J. L. (1965). Predation and relative helmet size in cyclomorphic Daphnia. Proceedings of the National Academy of Sciences USA 53, 119-126.

651 Buntrock, L., Marec, F., Krueger, S. \& Traut, W. (2012). Organ growth without cell 652

653 Cavalier-Smith, T. (1978). Nuclear volume control by nucleoskeletal DNA, selections for cell 654 volume and cell growth rate and the solution of the DNA C-value paradox. Journal of $655 \quad$ Cell Science 34, 247-278.

656 Cavalier-Smith, T. (1982). Skeletal DNA and the evolution of genome size. Annual Review of 657 Biophysics and Bioengineering 11, 273-302.

658 Cheniclet, C., Rong, W. Y., Causse, M., Bolling, L., Frangne, N., Carde, J.-P. \& 659 Renaudin, J.-P. (2005). Cell expansion and endoreduplication show a large genetic 660 variability in pericarp and contribute strongly to tomato fruit growth. Plant Physiology $661 \quad$ 139, 1984-1994.

662 Chevalier, C., Nafati, M., Mathieu-Rivet, E., Bourdon, M., Frangne, N., Cheniclet, C., 663 Renaudin, J.-P., Gévaudant, F. \& Hernould, M. (2011). Elucidating the functional role of 664 endoreduplication in tomato fruit development. Annals of Botany 107, 1159-1169.

665 Colbourne, J. K., Pfrender, M. E., Gilbert, D., Thomas, W. K., Tucker, A., Oakley, T. H., et 
al. (2011). The ecoresponsive genome of Daphnia pulex. Science 331, 555-561.

667 Comai, L. (2005). The advantages and disadvantages of being polyploid. Nature Reviews $668 \quad$ Genetics 6, 836-846.

669 Day, S. \& Lawrence, P. (2000). Measuring dimensions: the regulation of size and shape. $670 \quad$ Development 127, 2977-2987.

671 De Veylder, L., Larkin, J. C. \& Schnittger, A. (2011). Molecular control and function of 672 endoreplication in development and physiology. Trends in Plant Science 16, 624-634.

673 Dewhurst, S. M., McGranahan, N., Burrell, R. A., Rowan, A. J., Grönroos, E., Endesfelder, D., 674 Joshi, T., Mouradov, D., Gibbs, P., Ward, R. L., Hawkins, N. J., Szallasi, Z., Sieber, O. 675 M. \& Swanton, C. (2014). Tolerance of whole-genome doubling propagates 676 chromosomal instability and accelerates cancer genome evolution. Cancer Discovery 4 , 677 $175-185$.

678 Drummond-Barbosa, D. \& Spradling, A. C. (2001). Stem cells and their progeny respond to 679 nutritional changes during Drosophila oogenesis. Developmental Biology 231, 265-278. 680 Dufresne, F. \& Hebert, P. D. N. (1995). Polyploidy and clonal diversity in an arctic cladoceran. $681 \quad$ Heredity $\mathbf{7 5}, 45-53$.

682 Edgar, B. A. \& Orr-Weaver, T. L. (2001). Endoreplication cell cycles: more for less. Cell 105, $683 \quad 297-306$.

684 Edgar, B. A., Zielke, N. \& Gutierrez, C. (2014). Endocycles: a recurrent evolutionary 685 innovation for post-mitotic cell growth. Nature Reviews Cell Biology 15, 197-210.

686 Erbrich, P. (1965). Über Endopolyploidie und Kernstrukturen in Endospermhaustorien. 687 Österreichische Botanische Zeitschrift 112, 197-262. 
688 Fankhauser, G. (1945). The effects of changes in chromosome number on amphibian 689 development. The Quarterly Review of Biology 20, 20-78.

690 Fankhauser, G. \& Humphrey, R. R. (1952). The rare occurrence of mitosis without spindle apparatus ("colchicine mitosis") producing endopolyploidy in embryos of the axolotl.

693 Flemming, A. J., Shen, Z. Z., Cunha, A., Emmons, S. W. \& Leroi, A. M. (2000). Somatic polyploidization and cellular proliferation drives body size evolution in nematodes. Proceedings of the National Academy of Sciences USA 75, 5285-5290.

696 Fox, D. T., Gall, J. G. \& Spradling, A. C. (2010). Error-prone polyploid mitosis during normal Drosophila development. Genes \& Development 24, 2294-2302.

698 Galbraith, D. W., Harkins, K. R. \& Knapp, S. (1991). Systematic endopolyploidy in Arabidopsis thaliana. Plant Physiology 96, 985-989.

700 Ganot, P. \& Thompson, E. M. (2002). Patterning through differential endoreduplication in 701 epithelial organogenesis of the chordate, Oikopleura dioica. Developmental Biology 252, 59-71.

703 Gegas, V. C., Wargent, J. J., Pesquet, E., Granqvist, E., Paul, N. D. \& Doonan, J. H. (2014).

704 Endopolyploidy as a potential alternative adaptive strategy for Arabidopsis leaf size

705 variation in response to UV-B. Journal of Experimental Botany 65, 2757-2766.

706 Gonzalez, N., Gévaudant, F., Hernould, M., Chevalier, C. \& Mouras, A. (2007). The cell cycleassociated protein kinase WEE1 regulates cell size in relation to endoreduplication in developing tomato fruit. The Plant Journal 51, 642-655.

709 Gregory, T. R. (2005). Genome size evolution in animals. In The Evolution of the Genome (ed T.

$710 \quad$ R. Gregory), pp. 3-87. Elsevier, Oxford. 
711 Gregory, T. R. \& Hebert, P. D. N. (1999). The modulation of DNA content: proximate causes 712 and ultimate consequences. Genome Research 9, 317-324.

713 Gregory, T. R. \& Shorthouse, D. P. (2003). Genome sizes of spiders. Journal of Heredity 94, $714 \quad 285-290$.

715 Griffiths, P. D., Ougham, H. J. \& Jones, R. N. (1994). Genotypic and environmental effects 716 on endopolyploidy in the epidermal tissues of Lolium perenne L. and Lolium multiflorum $717 \quad$ Lam. New Phytologist 128, 399-345.

718 Hardie, D. C., Gregory, T. R. \& Hebert, P. D. (2002). From pixels to picograms: a beginners' 719 guide to genome quantification by Feulgen image analysis densitometry. Journal of $720 \quad$ Histochemistry \& Cytochemistry 50, 735-749.

721 Henery, C. C., Bard, J. B. L. \& Kaufman, M. H. (1992). Tetraploidy in mice, embryonic cell 722 number, and the grain of the developmental map. Developmental Biology 152, 233-241.

723 Hessen, D. O., Jeyasingh, P. D., Neiman, M. \& Weider, L. J. (2010). Genome streamlining and 724 the elemental costs of growth. Trends in Ecology and Evolution 25, 75-80.

725 Hessen, D. O., Daufresne, M. \& Leinaas, H. P. (2013). Temperature-size relations from the 726 cellular-genomic perspective. Biological Reviews 88, 476-489.

727 Jalal, M., Andersen, T. \& Hessen, D. O. (2015). Temperature and developmental responses of 728 body and cell size in Drosophila; effects of polyploidy and genome configuration. 729 Journal of Thermal Biology 51, 1-14.

730 Jalal, M., Wojewodzic, M. W., Laane, C. M. M. \& Hessen, D. O. (2013). Larger Daphnia at 731 lower temperature: A role for cell size and genome configuration? Genome 56, 511-519.

732 John, P. C. L. \& Qi, R. (2008). Cell division and endoreduplication: doubtful engines of 733 vegetative growth. Trends in Plant Science 13, 121-127. 
734 Johnson, E. C. \& White, M.P. (2009). Stressed-out insects: Hormonal actions and behavioral 735 modifications. In Hormones, Brain and Behavior (eds D. W. Pfaff, Arnold, A. P., 736 Fahrbach, S. E., Etgen, A. M. \& Rubin, R.T.), pp 1069-1096. Academic Press, San $737 \quad$ Diego.

738 Jovtchev, G., Barow, M., Meister, A. \& Schubert, I. (2007). Impact of environmental and 739 endogenous factors on endopolyploidization in angiosperms. Environmental and $740 \quad$ Experimental Botany 60, 404-411.

741 King, K. C., Seppälä, O. \& Neiman, M. (2012). Is more better? Polyploidy and parasite 742 resistance. Biology Letters 8, 598-600.

743 Kikuchi, S. (1983). The fine structure of the gill epithelium of a fresh-water flea, Daphnia 744 magna (Crustacea: Phyllopoda) and changes associated with acclimation to various 745 salinities. Cell and Tissue Research 229, 253-268.

746 Korpelainen, H., Ketola, M. \& Hietala, J. (1997). Somatic polyploidy examined by flow 747 cytometry in Daphnia. Journal of Plankton Research 19, 2031-2040.

748 Lasek, R. J. \& Dower, W. J. (1971). Aplysia californica: analysis of nuclear DNA in 749 individual nuclei of giant neurons. Science 172, 278-280.

750 Lee, H. O., Davidson, J. M. \& Duronio, R. J. (2009). Endoreplication: polyploidy with $751 \quad$ purpose. Genes \& Development 23, 2461-2477.

752 Leggatt, R. A. \& Iwama, G. K. (2003). Occurrence of polyploidy in the fishes. Reviews in Fish $753 \quad$ Biology and Fisheries 13, 237-246.

754 Leiva-Neto, J. T., Grafi, G., Sabelli, P. A., Dante, R. A., Woo, Y. M., Maddock, S., 
Gordon-Kamm, W. J. \& Larkins, B. A. (2004). A dominant negative mutant of cyclindependent kinase A reduces endoreduplication but not cell size or gene expression in maize endosperm. The Plant Cell 16, 1854-1869.

758 Leslie, M. (2014). Strength in numbers? Science 343, 725-727.

759 Li, Y.-F., Chen, X.-Y., Zhang, C.-D., Tang, X.-F., Wang, L., Liu, T.-H., Pan, M.-H. \& Lu, C.

760 (2015). Effects of starvation and hormones on DNA synthesis in silk gland cells of the silkworm, Bombyx mori. Insect Science, in press. DOI: 10.1111/1744-7917.12199.

762 López-Juez, E., Dillon, E., Magyar, Z., Khan, S., Hazeldine, S., de Jager, S. M., Murray, J. A.,

763 Beemster, G. T., Bögre, L. \& Shanahan, H. (2008). Distinct light-initiated gene

764 expression and cell cycle programs in the shoot apex and cotyledons of Arabidopsis. The

$765 \quad$ Plant Cell 20, 947-968.

766 Losick, V. P., Fox, D. T. \& Spradling, A. C. (2013). Polyploidization and cell fusion contribute

767 to wound healing in the adult Drosophila epithelium. Current Biology 23, 2224-2232.

768 Lozano, E., Saeg, A. E., Flemming, A. J., Cunha, A. \& Leroi, A. M. (2006). Regulation of

769 growth by ploidy in Caenorhabditis elegans. Current Biology 16, 493-498.

770 Lynch, M. (2007). The Origins of Genome Architecture. Sinauer Associates, Sunderland.

771 Mable, B. K. (2004). 'Why polyploidy is rarer in animals than in plants:' myths and 772 mechanisms. Biological Journal of the Linnean Society 82, 453-466.

773 Mable, B. K., Alexandrou, M. A. \& Taylor, M. I. (2011). Genome duplication in amphibians and 774 fish: an extended synthesis. Journal of Zoology 284, 151-182.

775 Mandrioli, M., Mola, L., Cuoghi, B. \& Sonetti, D. (2010). Endoreplication: A molecular trick 776 during animal neuron evolution. The Quarterly Review of Biology 85, 159-169. 
777 Massonnet, C., Tisné, S., Radziejwoski, A., Vile, D., De Veylder, L., Dauzat, M. \&

778 Granier, C. (2011). New insights into the control of endoreduplication:

779 endoreduplication could be driven by organ growth in Arabidopsis leaves. Plant

$780 \quad$ Physiology 157, 2044-2055.

781 Mayfield-Jones, D., Washburn, J. D., Arias, T., Edger, P. P., Pires, J. C. \& Conant, G. C. (2013).

782 Watching the grin fade: Tracing the effects of polyploidy on different evolutionary time

783 scales. Seminars in Cell and Developmental Biology 24, 320-331.

784 McKinnon, A. H. (2013). Differential display: Moving towards unraveling the genetic basis of

785 predator induced defenses in Daphnia lumholtzi. BSc Honours Thesis, Mount Allison

$786 \quad$ University, Sackville.

787 Melaragno, J. E., Mehrotra, B. \& Coleman, A. W. (1993). Relationship between

788 endopolyploidy and cell size in epidermal tissue of Arabidopsis. The Plant Cell 5, 1661-

$789 \quad 1668$.

790 Mendell, J. E., Clements, K. D., Choat, J. H. \& Angert, E. R. (2008). Extreme polyploidy in a

791 large bacterium. Proceedings of the National Academy of Sciences USA 105, 6730-6734.

792 Nagl, W. (1976). DNA endoreplication and polyteny understood as evolutionary strategies.

$793 \quad$ Nature 261, 614-615.

794 Nagl, W. (1978). Endopolyploidy and Polyteny in Differentiation and Evolution. North

$795 \quad$ Holland Publishing Company, Amsterdam.

796 Neiman, M., Kay, A. D. \& Krist, A. M. (2013a). Can resource costs of polyploidy

797 provide an advantage to sex? Heredity 110, 152-159.

798 Neiman, M., Kay, A. D. \& Krist, A. M. (2013b). Sensitivity to phosphorus limitation

799 increases with ploidy level in a New Zealand snail. Evolution 67, 1511-1517. 
800 Neiman, M., Theisen, K., Mayry, M. E. \& Kay, A. D. (2009). Can phosphorus limitation 801 contribute to the maintenance of sex? A test of a key assumption. Journal of 802 Evolutionary Biology 22, 1359-1363.

803 Neiman, M., Paczesniak, D., Soper, D. M., Baldwin, A. T. \& Hehman, G. (2011). Wide 804 variation in ploidy level and genome size in a New Zealand freshwater snail with 805 coexisting sexual and asexual lineages. Evolution 65, 3202-3216.

806 Olmo, E. (1983). Nucleotype and cell size in vertebrates: A review. Basic and Applied $807 \quad$ Histochemistry 27, 227-256.

808 Parfrey, L. W., Lahr, D. J. G. \& Katz, L. A. (2008). The dynamic nature of eukaryotic 809 genomes. Molecular Biology and Evolution 25, 787-794.

810 Parisod, C., Holderegger, R. \& Brochmann, C. (2010). Evolutionary consequences of 811 autopolyploidy. New Phytologist 186, 5-17.

812 Perdrix-Gillot, S. (1979). DNA synthesis and endomitoses in the giant nuclei of the silk gland of 813 Bombyx mori. Biochimie 61, 171-204.

814 Rasch, E. M. (2004). Feulgen-DNA cytophotometry for estimating C values. In: Drosophila 815 Cytogenetics Protocols. Methods in Molecular Biology 247, 163-201.

816 Rasch, E. M. \& Connelly, B. A. (2005). Genome size and endonuclear DNA replication in 817 spiders. Journal of Morphology 265, 209-214.

818 Rasch, E. M. \& Wyngaard, G. A. (2008). Endopolyploidy in cyclopoid copepods. Journal of 819 Crustacean Biology 28, 412-416.

820 Roth, G., Blanke, J. \& Wake, D. B. (1994). Cell size predicts morphological complexity in the 821 brains of frogs and salamanders. Proceedings of the National Academy of Sciences USA $822 \quad 91,4796-4800$. 
823 Roth, G., Blanke, J. \& Wake, D. B. (1995). Brain size and morphology in miniaturized

824 plethodontid salamanders. Brain Behavior and Evolution 45, 84-95.

825 Santamaria, P. (1983). Analysis of haploid mosaics in Drosophila. Developmental

826 Biology 96, 285-295.

827 Santini, F., Harmon, L. J., Carnevale, G. \& Alfaro, M. E. (2009). Did genome duplication drive 828 the origin of teleosts? A comparative study of diversification in ray-finned fishes. BMC $829 \quad$ Evolutionary Biology 9, 194.

830 Scholes, D. R. \& Paige, K. N. (2011). Chromosomal plasticity: mitigating the impacts of 831 herbivory. Ecology 92, 1691-1698.

832 Scholes, D. R. \& Paige, K. N. (2014). Plasticity in ploidy underlies plant fitness compensation to 833 herbivore damage. Molecular Ecology 23, 4862-4870.

834 Scholes, D. R., Suarez, A. V. \& Paige, K. N. (2013). Can endopolyploidy explain body size 835 variation within and between castes in ants? Ecology and Evolution 3, 2128-2137.

836 Scholes, D. R., Suarez, A. V., Smith, A. A., Johnston, J. S. \& Paige, K. N. (2014). Organ837 specific patterns of endopolyploidy in the giant ant Dinoponera australis. Journal of $838 \quad$ Hymenopteran Research 37, 113-126.

839 Seehausen, O., Butlin, R. K., Keller, I., Wagner, E., Boughman, J. W., Hohenlohe, P. et al. 840 (2014). Genomics and the origin of species. Nature Reviews Genetics 15, 176-192.

841 Sehnal, F. \& Sutherland, T. (2008). Silks produced by insect labial glands. Prion 2, 145-153.

842 Selmecki, A. M., Y. E. Maruvka, P. A. Richmond, M. Guillet, N. Shoresh, A. L. Sorenson, S.

843 De, R. Kishnoy, F. Michor, R. Dowell, and D. Pellman. (2015) Polyploidy can drive 844 rapid adaptation in yeast. Nature 519, 349-352.

845 Sher, N., Von Stetina, J. R., Bell, G. W., Matsuura, S., Ravid, K. \& Orr-Weaver, T. L. 
(2013). Fundamental differences in endoreplication in mammals and Drosophila

847

848

849

850

851

852

853

854

855

856

857

858

859

860

861

862

863

864

865

866

867

revealed by analysis of endocycling and endomitotic cells. Proceedings of the National Academy of Sciences USA 110, 9368-9373.

Šmarda, P., Hejcman, M., Březinová, A., Horová, L., Steigerová, H., Zedek, F., Bureš, P., Hejcmanová, P. \& Schellberg, J. (2013). Effect of phosphorus availability on the selection of species with different ploidy levels and genome sizes in a long-term grassland fertilization experiment. New Phytologist 200, 911-921.

Soltis, D. E., Albert, V. A., Leebens-Mack, J., Bell, C. D., Paterson, A. H., Zheng, C., Sankoff, D., dePamphilis, C. W., Wall, P. K. \& Soltis, P. S. (2009). Polyploidy and angiosperm diversification. American Journal of Botany 96, 336-348.

Soltis, P. S., Liu, X., Marchant, D. B., Visger, C. J. \& Soltis, D. E. (2014). Polyploidy and novelty: Gottlieb's legacy. Philosophical Transactions of the Royal Society of London B 369, 20130351.

Sterba, G. (1956). Die neurosekretorischen Zellgruppen einiger Cladoceren (Daphnia pulex und magna, Simocephalus vetulus). Zoologische Jahrbücher. Abteilung für Anatomie und Ontogenie der Tiere 76, 303-310.

Sterba, G. (1957). Die Riesenzellen der Daphnien-Oberlippe. Zeitschrift für Zellforschung und mikroskopische Anatomie 47, 198-213.

Sterner, R. W. \& Elser, J. J. (2002). Ecological Stoichiometry: The Biology of Elements from Molecules to the Biosphere. Princeton University Press, Princeton.

Sugimoto-Shirasu, K. \& Roberts, K. (2003). "Big it up": endoreduplication and cell-size control in plants. Current Opinion in Cell Biology 6, 544-553. 
868 Szaro, B. G., Tompkins, R. \& Szaro, B. G. (1987). Effect of tetraploidy on dendritic 869 branching in neurons and glial cells of the frog, Xenopus laevis. Journal of $870 \quad$ Comparative Neurology 258, 304-316.

871 Vanneste, K., Maere, S. \& Van de Peer, Y. (2014). Tangled up in two: a burst of genome 872 duplications at the end of the Cretaceous and the consequences for plant evolution. 873 Philosophical Transactions of the Royal Society of London B 369, 20130353.

874 Vernon, J. A. \& Butsch, J. (1957). Effect of tetraploidy on learning and retention in the 875 salamander. Science 125, 1033-1034.

876 Vinogradov, A. E., Anatskaya, O. V. \& Kudryavtsev, B. N. (2001). Relationship of 877 hepatocyte ploidy levels with body size and growth rate in mammals. Genome 44, 350$878 \quad 360$.

879 Weiss, L. C., Tollrian, R., Herbert, Z. \& Laforsch, C. (2012). Morphology of the Daphnia 880 nervous system: A comparative study on Daphnia pulex, Daphnia lumholtzi, and 881 Daphnia longicephala. Journal of Morphology 273, 1392-1405.

882 Wilson, W. W. \& Roach, P. J. (2002). Nutrient-regulated protein kinases in budding yeast. Cell $883 \quad \mathbf{1 1 1}, 155-158$.

884 Xia, Q., Guo, Y., Zhang, Z., Li, D., Xuan, Z., Li, Z., Dai, F. et al. (2009). Complete 885 resequencing of 40 genomes reveals domestication events and genes in silkworm $886 \quad$ (Bombyx). Science 326, 433-436.

887 Yamagishi M., Ito, E. \& Matsuo, R. (2011). DNA endoreplication in the brain neurons during 888 body growth of an adult slug. Journal of Neuroscience 31, 5596-5604. 
889 Yin, L., Gater, S. T. \& Karrer, K. M. (2010). A developmentally regulated gene, ASI2, is

890 required for endocycling in the macronuclear anlagen of Tetrahymena. Eukaryotic Cell $\mathbf{9}$, $891 \quad 1343-1353$.

892 Zhang, C.-D., Li, F.-F., Chen, X.-Y., Huang, M.-H., Zhang, J., Cui, H., Pan, M.-H. \& Lu, C. 893 (2012). DNA replication events during larval silk gland development in the silkworm, 894 Bombyx mori. Journal of Insect Physiology 58, 974-978.

895

896 Figure Legends

897

898 Figure 1.

899 Endopolyploidy in adult female Daphnia lumholtzi from laboratory cultures. Six Feulgen-stained 900 tissues showing ploidy level ranges: a. head epidermis; b. labrum; c. appendage with exopodite 901 (2C), epipodite (8C), and lipid cells (64-256C); d. thoracic epidermis; e digestive tract; f. shell 902 gland. All scale bars indicate $50 \mu \mathrm{m}$. 


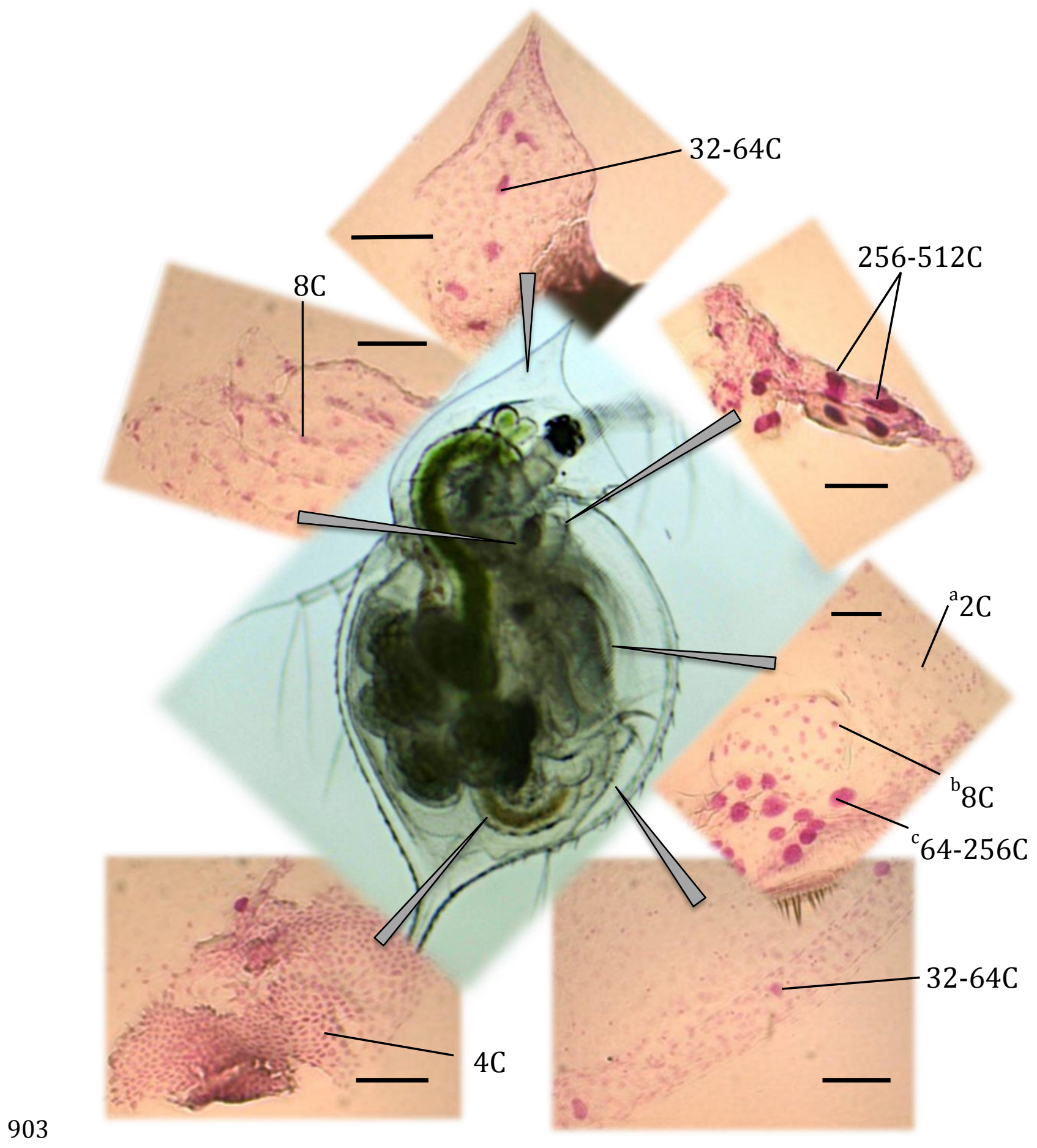

